\title{
Error Structure of Multiparameter Radar and Surface Measurements of Rainfall Part II: X-Band Attenuation
}

\author{
V. CHANDRASEKAR AND V. N. BRINGI \\ Department of Electrical Engineering, Colorado State University. Ft. Collins, Colorado
}

(Manuscript received 26 June 1987, in final form 4 April 1988)

ABSTRACT

\begin{abstract}
In Part II of this study, simulations of multiparameter radar observables to include $\mathrm{X}$-band specific attenuation $(A)$ are performed in order to study the relationship between $A, Z$, and $Z_{\mathrm{DR}}$. We also compute the triplet ( $A$, $Z, Z_{\mathrm{DR}}$ ) from simulations of disdrometer raindrop spectra. As in Part $\mathrm{I}$, our simulations include the fluctuations due to both measurement errors and physical variations of the gamma raindrop spectra parameters $\left(N_{0}, D_{0}\right.$, $m$ ). We examine the correlation between $(A / Z)$ and $Z_{D R}$ derived from both disdrometer and radar simulations, and show that the disdrometer-based data yields a negative correlation $(\sim-0.9)$ between $(A / Z)$ and $Z_{\mathrm{DR}}$, whereas for radar data the correlation $\approx 0$. We emphasize that these correlations are due only to measurement fluctuations, and not to physical variations. The lange magnitude for the negative correlation compresses the scatter in plots of $(A / Z)$ versus $Z_{\mathrm{DR}}$ based on disdrometer RSD samples, whereas the same scatter plots using multiparameter radar data show very large scatter. We also simulate $A, Z$ and $Z_{\mathrm{DR}}$ from three separate disdrometers (all sampling the same gamma RSD) and show that the scatter is more realistic and much larger than when using a single disdrometer.
\end{abstract}

\section{Introduction}

Part I of this two-part paper dealt with the error structure of differential reflectivity and surface measurements of rainfall. In this part we continue these studies by focusing on $\mathrm{X}$-band attenuation due to rain and consider the triplet of measurements $\left(A, Z, Z_{\mathrm{DR}}\right)$ corresponding to specific attenuation $\left(\mathrm{dB} \mathrm{km}^{-1}\right)$, radar reflectivity and differential reflectivity, respectively. The physical basis for attenuation-rainfall relationships has been extensively studied; see, for example, Atlas and Ulbrich $(1974,1977)$, and the review article by Oguchi (1983), which includes an extensive reference list. More recently, radar measurements of $Z$ and $Z_{\mathrm{DR}}$ have been used to predict rain attenuation in the $10-30 \mathrm{GHz}$ range (Goddard and Cherry 1984; Leitao and Watson 1984), while Bringi et al. (1986) have directly intercompared path-averaged X-band attenuation predicted by $Z$ and $Z_{D R}$ (and a gamma raindrop size distribution model) with path averaged $X$-band attenuation measured by the dual-frequency ratio technique (Eccles and Mueller 1973; Aydin et al. 1983; Tuttle and Rinehart 1983). However, there are only two radars capable of making the triplet of measurements $\left(A, Z, Z_{\mathrm{DR}}\right)$ : the National Center for Atmospheric Research CP-2 radar and the Illinois State Water Survey CHILL radar.

Atlas et al. (1984) have computed the triplet $(A, Z$,

Corresponding author address: Dr. V. N. Bringi, Dept. of Electrical Engineering, Colorado State University, Ft. Collins, CO 80523.
$Z_{D R}$ ) from raindrop size distribution (RSD) samples obtained from momentum disdrometers (Joss and Waldvogel 1969), and show scatter plots of rain rates derived using multiparameter methods, e.g., $(Z, A)$, $\left(Z, Z_{\mathrm{DR}}\right)$ or $\left(A, Z, Z_{\mathrm{DR}}\right)$ versus disdrometer measured rain rates. Their "simulations" of radar observables showed that the absolute average deviation (AAD) between multiparameter-deduced rain rates and "actual" rain rates could be made extremely small ( $\leqslant 5 \%)$ and much less than single-parameter methods (e.g., $Z-R$ relations) that gave AADs of $\sim 30 \%$. In this paper, using analytical techniques and simulations, we examine the relationship between $A, Z$, and $Z_{\mathrm{DR}}$ as measured by radar and as computed from disdrometer RSD samples. As explained in Part I, it is our intent to simulate the fluctuations in the observables so that both statistical and physical variations can be included.

Figure 1a shows a scatter plot of $A / Z$ versus $Z_{D R}$ computed from disdrometer RSD samples by Aydin et al. (1983). The scatter in Fig. 1a is very small and clearly shows the mean relationship between $A / Z$ and $Z_{\mathrm{DR}}$. Figure $1 \mathrm{~b}$ shows $\mathrm{CP}-2$ radar measurements from convective rainshafts taken on 13 June 1984 -near Boulder, Colorado; see Bringi et al. (1986) for details regarding these measurements. In Fig. 1b, the dualfrequency algorithm developed by Tuttle and Rinehart (1983) is used to estimate path-averaged X-band specific attenuation. Figure $1 \mathrm{~b}$ shows the scatter plot of $A / Z$ versus $Z_{\mathrm{DR}}$, where each observable is obtained from radar data. Clearly the scatter in Fig. $1 \mathrm{~b}$ is much larger than in Fig. la, and it is desirable to understand 

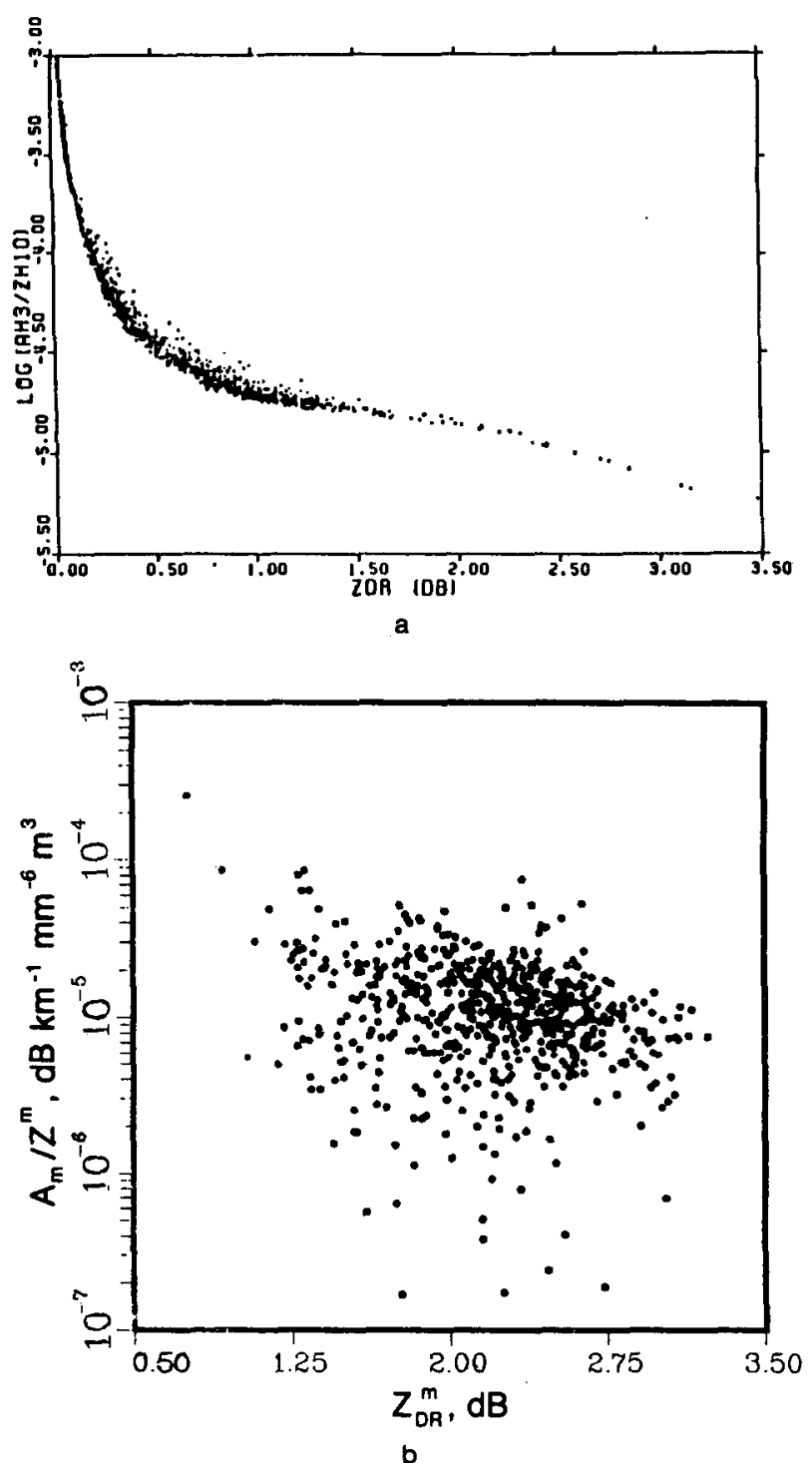

FIG. 1. a. Scatter plot of $(A / Z)$ versus $Z_{\mathrm{DR}}$ based on disdrometer RSD samples. Note that $\mathrm{AH} 3 \equiv A$ and $\mathrm{ZH} 10 \equiv Z$. Units of $A$ are $\mathrm{dB} \mathrm{km} \mathrm{km}^{-1}$ while $Z$ is in $\mathrm{mm}^{6} \mathrm{~m}^{-3}$. Figure reproduced from Aydin et al. (1983). b. Scatter plot of $(A / Z)$ versus $Z_{\mathrm{DR}}$ in which all quantities were measured by the CP.2 radar system on 13 June 1984 near Boulder, Colorado. Radar data is from convective rainshafts. Figure reproduced from Bringi et al. (1986).

the sources of this scatter, i.e., performing an analysis of variance on the data. As shown in Part I and in the current work, the correlation between the triplet of observables $\left(A, Z, Z_{\mathrm{DR}}\right)$ plays an important role in determining the scatter between $A / Z$ and $Z_{\mathrm{DR}}$.

In section 2 we derive a mean relationship between $A / Z$ and $Z_{\mathrm{DR}}$ for a gamma RSD model. In section 3 we analytically derive the correlation between $A / Z$ and $Z_{\mathrm{DR}}$, assuming the measurements are obtained with a (fixed sampling volume) disdrometer sampling gamma RSDs. We also derive analytically the covariance be- tween radar $Z$ and $Z_{\mathrm{DR}}$ for logarithmic receivers. In section 4 we simulate the radar observables $A, Z$, and $Z_{\mathrm{DR}}$ in order to study their correlations. In section 5 we simulate $A, Z$, and $Z_{\mathrm{DR}}$ using RSD simulations from three independent disdrometers all sampling the same mean rainfall. The resultant scatter is compared with the compression of the scatter in Fig. 1a and in the data of the type presented by Atlas et al. (1984).

\section{Prediction of $X$-band specific attenuation}

We use a gamma RSD model to derive the mean relationship between $A / Z$ and $Z_{\mathrm{DR}}$. (See section 2 of Part I for a discussion of the gamma RSD model, with reflectivity and $Z_{\mathrm{DR}}$ defined by (4) and (7), respectively.) Specific attenuation $(A)$ is defined by,

$$
A=0.4343 \int \sigma_{\mathrm{ext}}(D) N(D) d D \mathrm{~dB} \mathrm{~km}^{-1},
$$

where $\sigma_{\text {ext }}(D)$ is the $\mathrm{X}$-band extinction cross section of a raindrop of diameter $D$ and $N(D)$ is the gamma RSD defined by (2) of Part I. The gamma parameters $\left(N_{0}, D_{0}, m\right)$ are varied over the entire range, representative of natural rainfall (Ulbrich 1983). For each triplet of values $\left(N_{0}, D_{0}, m\right)$ we compute $A, Z$, and $Z_{\mathrm{DR}}$; Fig. 2 shows a scatter plot of $A^{\text {sd }} / Z^{\text {sd }}$ versus $Z_{\mathrm{DR}}^{\text {sd }}$ in which the superscript "sd" stands for observables calculated from gamma RSDs. The scatter in Fig. 2 is negligible, and the curve implies that, in principle, measurements of $Z$ and $Z_{\mathrm{DR}}$ should enable us to estimate $A$ accurately (assuming, of course, that the gamma RSD model is valid); see Jameson (1983), Aydin et al. (1983).

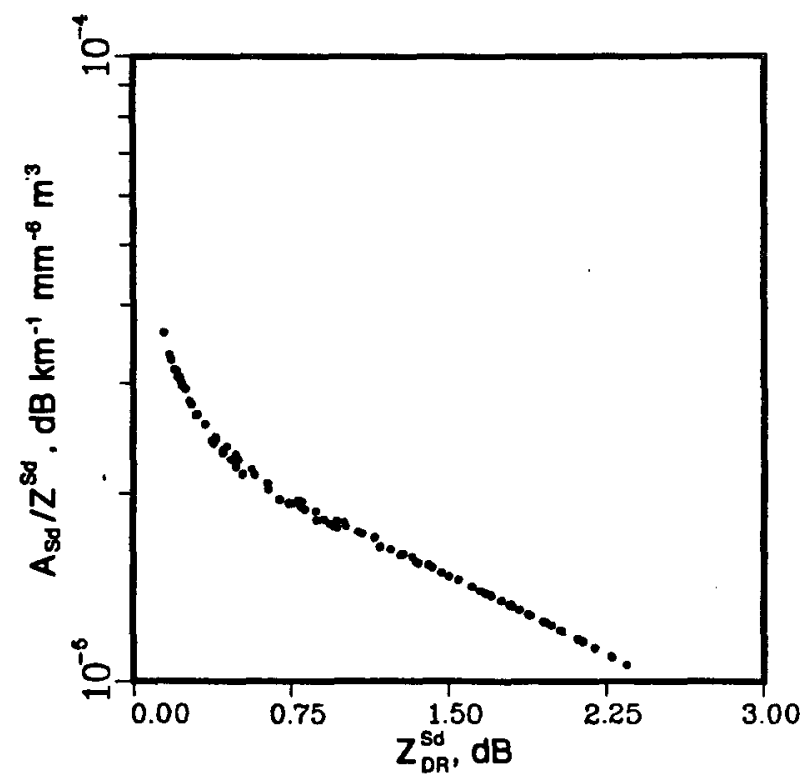

FIG. 2. Scatter plot of $\left(A^{\text {sd }} / Z^{\text {sd }}\right)$ versus $Z_{\mathrm{DR}}^{\text {sd }}$ for gamma RSDs. Each point represents a triplet of values $\left(N_{0}, D_{0}, m\right)$, which vary over a wide range. 


\section{Correlation between observables}

\section{a. Estimates based on disdrometer}

In section 5 of Part I we derived the correlation coefficient between $Z$ and $Z_{\mathrm{DR}}$ where both quantities are calculated from disdrometer data, the disdrometer samples coming from a gamma RSD model.

In this section we derive the correlation coefficient between $A / Z$ and $Z_{\mathrm{DR}}$. The $[A / Z]$ estimates from the disdrometer can be written as

$$
[\hat{A} / \hat{Z}]=\frac{0.4343 \sum_{i=1}^{n} \sigma_{\mathrm{ext}}(D)}{C^{*} \sum_{i=1}^{n} \sigma_{H H}(D)}=\frac{P}{X}
$$

where $\sigma_{H H}(D)$ is the S-band radar cross section at horizontal polarization; $C^{*}$ and $\hat{Z}_{\mathrm{DR}}(=X / Y)$ are defined in (24) and Eq. (22a) of Part I, respectively.

The covariance between $[\hat{A} / \hat{Z}]$ and $\hat{Z}_{\mathrm{DR}}$ can be written as

$$
\operatorname{cov}\left[\hat{A} / \hat{Z}, \hat{Z}_{\mathrm{DR}}\right]=\operatorname{cov}\left[\frac{P}{X}, \frac{X}{Y}\right]
$$

Expanding $\frac{P}{X}$ and $\frac{X}{Y}$ in two dimensional series about their statistical expectations we get

$$
\begin{aligned}
& \frac{P}{X} \approx \frac{E(P)}{E(X)}\left[1+\frac{(P-E(P))}{E(P)}-\frac{(X-E(X))}{E(X)}\right] \\
& \frac{X}{Y} \approx \frac{E(X)}{E(Y)}\left[1+\frac{(X-E(X))}{E(X)}-\frac{Y-E(Y)}{E(Y)}\right],
\end{aligned}
$$

where $E(\quad)$ represents the statistical expectation.
Substituting (4) in (3) and simplifying we get

$$
\begin{aligned}
\operatorname{cov}\left[\frac{P}{X},\right. & \left.\frac{X}{Y}\right] \approx \frac{E(P)}{E(Y)}\left[\frac{\operatorname{cov}(P, X)}{E(P) E(X)}\right. \\
& \left.-\frac{\operatorname{cov}(P, Y)}{E(P) E(Y)}-\frac{\operatorname{var}(X)}{E^{2}(X)}+\frac{\operatorname{cov}(X, Y)}{E(X) E(Y)}\right] .
\end{aligned}
$$

In the above equation, the abbreviations "cov" and "var" indicate covariance and variance, respectively. Using techniques discussed in section 4 of Part I we can write the variance of $[\hat{A} / \hat{Z}]$ and $\hat{Z}_{\mathrm{DR}}$ as (Mood et al. 1974),

$$
\begin{aligned}
\operatorname{var}(A / Z) & =\left[\frac{E(P)}{E(X)}\right]^{2} \\
\times & {\left[\frac{\operatorname{var}(P)}{E^{2}(P)}+\frac{\operatorname{var}(X)}{E^{2}(X)}-2 \frac{\operatorname{cov}(P, X)}{E(P) E(X)}\right] }
\end{aligned}
$$

$$
\begin{aligned}
\operatorname{var}\left(\hat{Z}_{\mathrm{DR}}\right)^{=}=\left[\frac{E(X)}{E(Y)}\right]^{2} \\
\times\left[\frac{\operatorname{var}(X)}{E^{2}(X)}+\frac{\operatorname{var}(Y)}{E^{2}(Y)}-2 \frac{\operatorname{cov}(X, Y)}{E(X) E(Y)}\right] .
\end{aligned}
$$

In (5) and (6), all expectations, variances and covariances, have to be computed over two-fold distributions, namely the RSD and the distribution of the total number of samples. Using the methods discussed in section 5 of Part I paper we get the correlation between $\frac{\hat{A}}{\hat{Z}}$ and $\hat{Z}_{\mathrm{DR}}$ as

$\rho\left(\frac{\hat{A}}{\hat{Z}}, \hat{Z}_{\mathrm{DR}}\right)=\frac{\left[\frac{E\left(\sigma_{\mathrm{ext}} \sigma_{H H}\right)}{E\left(\sigma_{\mathrm{ext}}\right) E\left(\sigma_{H H}\right)}-\frac{E\left(\sigma_{\mathrm{ext}} \sigma_{V V}\right)}{E\left(\sigma_{\mathrm{ext}}\right) E\left(\sigma_{V V}\right)}-\frac{E\left(\sigma_{H H}^{2}\right)}{E^{2}\left(\sigma_{H H}\right)}+\frac{E\left(\sigma_{H H} \sigma_{V V}\right)}{E\left(\sigma_{H H}\right) E\left(\sigma_{V V}\right)}\right]}{\left[\left(\frac{E\left(\sigma_{\mathrm{ext}}^{2}\right)}{E^{2}\left(\sigma_{\mathrm{ext}}\right)}+\frac{E\left(\sigma_{H H}^{2}\right)}{E^{2}\left(\sigma_{H H}\right)}-2 \frac{E\left(\sigma_{\mathrm{ext}} \sigma_{H H}\right)}{E\left(\sigma_{\mathrm{ext}}\right) E\left(\sigma_{H H}\right)}\right)\left(\frac{E\left(\sigma_{H H}^{2}\right)}{E^{2}\left(\sigma_{H H}\right)}+\frac{E\left(\sigma_{V V}^{2}\right)}{E^{2}\left(\sigma_{V V}\right)}-2 \frac{E\left(\sigma_{H H} \sigma_{V V}\right)}{E\left(\sigma_{H H} \sigma_{V V}\right)}\right)\right]^{1 / 2}}$.

Equation (7) gives the correlation of $\left(\frac{\hat{A}}{\hat{Z}}\right)$ with $\hat{Z}_{\mathrm{DR}}$, and the results are shown in Fig. 3, where the correlation coefficient is plotted as a function of $D_{0}$ with $m$ as a parameter. We can see from Fig. 3 that the correlation coefficient is negative and typically takes values between -0.8 to -1 . This negative correlation is responsible for the reduction of the scatter in $[A / Z]$ versus $Z_{\mathrm{DR}}$ plots since the mean $[A / Z]$ decreases with increasing mean $Z_{D R}$; see also Fig. 2.

\section{b. Estimates based on radar}

In section 5 of Part I we showed that $Z$ and $Z_{D R}$ estimated from the disdrometer are correlated. In this section we show analytically that radar estimates of $Z$ and $Z_{\mathrm{DR}}$ are nearly uncorrelated for a logarithmic receiver.

A log receiver estimate of $Z_{\mathrm{DR}}$ is defined as (Bringi et al. 1983),

$$
\hat{Z}_{\mathrm{DR}}(\mathrm{dB})=\frac{1}{M} 10 \sum_{i=1}^{M} \log \left[\frac{p_{H}^{i}}{p_{V}^{i}}\right],
$$

where $M$ is the number of sample pairs, and $p_{H}{ }^{i}$ and $p_{V}{ }^{i}$ are the $i$ th power samples at $H$ and $V$ polarizations, respectively.

Reflectivity estimate is defined as

$$
\hat{Z}=\frac{1}{M} 10 \sum_{i=1}^{M} \log \left[p_{H}{ }^{i}\right]
$$




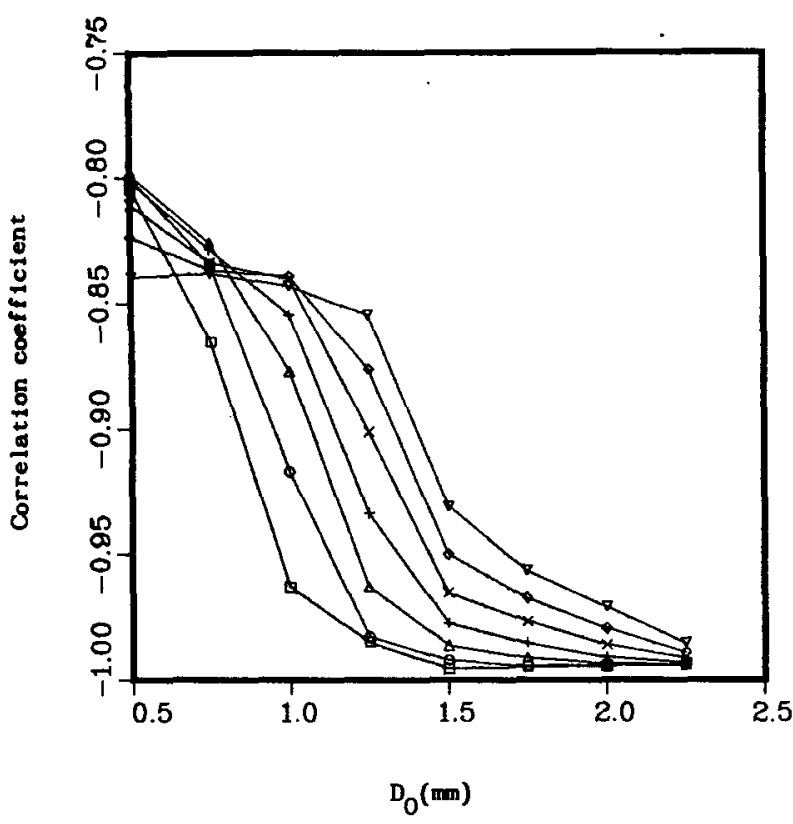

FIG. 3. Correlation coefficient between $(A / Z)$ and $Z_{\mathrm{DR}}$ obtained from disdrometer samples as a function of $D_{0}$ with $m$ as a parameter, $\square, m=0 ; \bigcirc, m=1 ; \Delta, m=2 ;+, m=3 ; \times, m=4 ; \diamond, m=5 ; \nabla$, $m=6$.

From ( 8 ) and (9) we can write the covariance between $\hat{Z}$ and $\hat{Z}_{\mathrm{DR}}$ as

$$
\begin{aligned}
& \operatorname{cov}\left(\hat{Z}, \hat{Z}_{\mathrm{DR}}\right) \\
& =\frac{18.86}{M^{2}} \operatorname{cov}\left[\sum_{i=1}^{M} \ln p_{H}{ }^{i}, \sum_{i=1}^{M} \ln \left[\frac{p_{H}{ }^{i}}{p_{V}{ }^{i}}\right]\right] \\
& =\frac{18.86}{M^{2}}\left\{\operatorname{var}\left[\sum_{i=1}^{M} \ln p_{H}{ }^{i}\right]\right. \\
& \left.\quad-\operatorname{cov}\left[\sum_{i=1}^{M} \ln p_{H}{ }^{i}, \sum_{i=1}^{M} \ln p_{V}{ }^{i}\right]\right\} .
\end{aligned}
$$

The above two covariances can be simplified as (Brockwell and Davis 1986)

$$
\begin{aligned}
\operatorname{var} & {\left[\sum_{i=1}^{M} \ln p_{H}^{i}\right]=\sum_{m=-(M-1)}^{(M-1)} } \\
& \times(M-|m|) \operatorname{cov}\left[\ln p_{H}^{i}, \ln p_{H}^{i+m}\right]
\end{aligned}
$$

and

$$
\begin{aligned}
\operatorname{cov}\left[\sum_{i=1}^{M} \ln \left[p_{H}{ }^{i}\right], \sum_{i=1}^{M} \ln \left[p_{V}{ }^{i}\right]\right]=\sum_{m=-(M-1)}^{(M-1)} \\
\times(M-|m|) \operatorname{cov}\left[\ln p_{H}{ }^{i}, \ln p_{V}{ }^{i+m}\right] .
\end{aligned}
$$

It can be shown that $\operatorname{var}\left[\ln p_{H, V}^{i}\right]=\frac{\pi^{2}}{6}$. Substituting these results into (10) we get

$$
\begin{aligned}
\operatorname{cov}\left[\hat{Z}, \hat{Z}_{\mathrm{DR}}\right] & =\frac{18.86}{M^{2}} \cdot \frac{\pi^{2}}{6}\left[\sum_{m=-(M-1)}^{(M-1)}\right. \\
& \left.\times(M-|m|)\left\{\rho_{H H}^{\log }(m)-\rho_{H V}^{\log }(m)\right\}\right],
\end{aligned}
$$

where $\rho^{\log }$ is the correlation between the $\log$ of the samples. We can see by comparing it with expressions in Chandrasekar et al. (1986) that $\operatorname{cov}\left[\hat{Z}, \hat{Z}_{\mathrm{DR}}\right]$ is onehalf the variance of $Z_{\mathrm{DR}}$ from $\log$ receivers. For alternate sampling, $\rho_{H H}(m)$ in (13) becomes $\rho_{H H}(2 m)$, and $\rho_{H V}(m)$ becomes $\rho_{H V}(2 m+1)$. Hence, the correlation coefficient $\rho\left(\hat{Z}, \hat{Z}_{\mathrm{DR}}\right)$ is given by

$$
\rho\left(\hat{Z}, \hat{Z}_{\mathrm{DR}}\right)=\frac{\frac{1}{2} \sigma_{Z_{\mathrm{DR}}}^{2}}{\sigma_{Z} \sigma_{Z_{\mathrm{DR}}}},
$$

where $\sigma_{Z}{ }^{2}$ is the variance of $\hat{Z}$ and $\sigma_{Z_{\mathrm{DR}}}^{2}$ is the variance of $\hat{Z}_{\mathrm{DR}}$. Equation (14) simplifies to

$$
\rho\left(\hat{Z}, \hat{Z}_{\mathrm{DR}}\right)=\frac{1}{2}\left(\frac{\sigma_{Z_{\mathrm{DR}}}}{\sigma_{Z}}\right) \text {. }
$$

Thus, $\rho\left(\hat{Z}, \hat{Z}_{\mathrm{DR}}\right)$ typically takes values around 0.08 . This indicates that for all practical purposes, we can consider $\hat{Z}$ and $\hat{Z}_{\mathrm{DR}}$ to be uncorrelated. On the other hand, estimates of $\dot{Z}$ and $\hat{Z}_{\mathrm{DR}}$ from disdrometer samples are highly correlated [see (27) and Fig. 2 of Part 1].

\section{Multiparameter radar simulations}

We refer to section 6 of Part I for a summary of methods related to simulating S-band reflectivity and $Z_{\mathrm{DR}}$; see also Chandrasekar et al. (1986) and Bringi et al. (1986) for details. The X-band power samples are simulated in the same manner as the S-band, since the two signals are independent. Specific attenuation over a range interval $\Delta r=\left(r_{2}-r_{1}\right)$ is simulated by using two uncorrelated X-band time series $\left(P_{X}\right)$ at ranges $r_{1}$ and $r_{2}$ with $10 \log \left[\bar{P}_{X}\left(r_{2}\right)\right]=10 \log \left[\bar{P}_{X}\left(r_{1}\right)\right]-2 \bar{A}(\Delta r)$, $\bar{A}$ being the average specific attenuation in the interval $\Delta r$. Uncorrelated, univariate time series are easily generated using Zrnić's (1975) procedure. Range averaging is simulated by generating independent time series, since signals from different ranges are independent. Table 1 gives a brief description of the assumptions used in the simulations, which are designed to be applicable to the $\mathrm{CP}-2$ radar.

TABLE 1. Parameters for radar simulation.

\begin{tabular}{lll}
\hline & \multicolumn{1}{c}{ S-band } & X-band \\
\hline Gaussian Doppler Spectrum Width & $3 \mathrm{~m} \mathrm{~s}^{-1}$ & $3 \mathrm{~m} \mathrm{~s}^{-1}$ \\
Pulse Repetition Time & $1 \mathrm{~ms}$ & $1 \mathrm{~ms}$ \\
Number of Samples & $128 \mathrm{H}, 128 \mathrm{~V}$ & 128 \\
Receiver Type & Logarithmic & Logarithmic \\
Range Averaging & 6 gates & 6 gates \\
Range Interval for Attenuation & - & $5 \mathrm{~km}$ \\
\hline
\end{tabular}



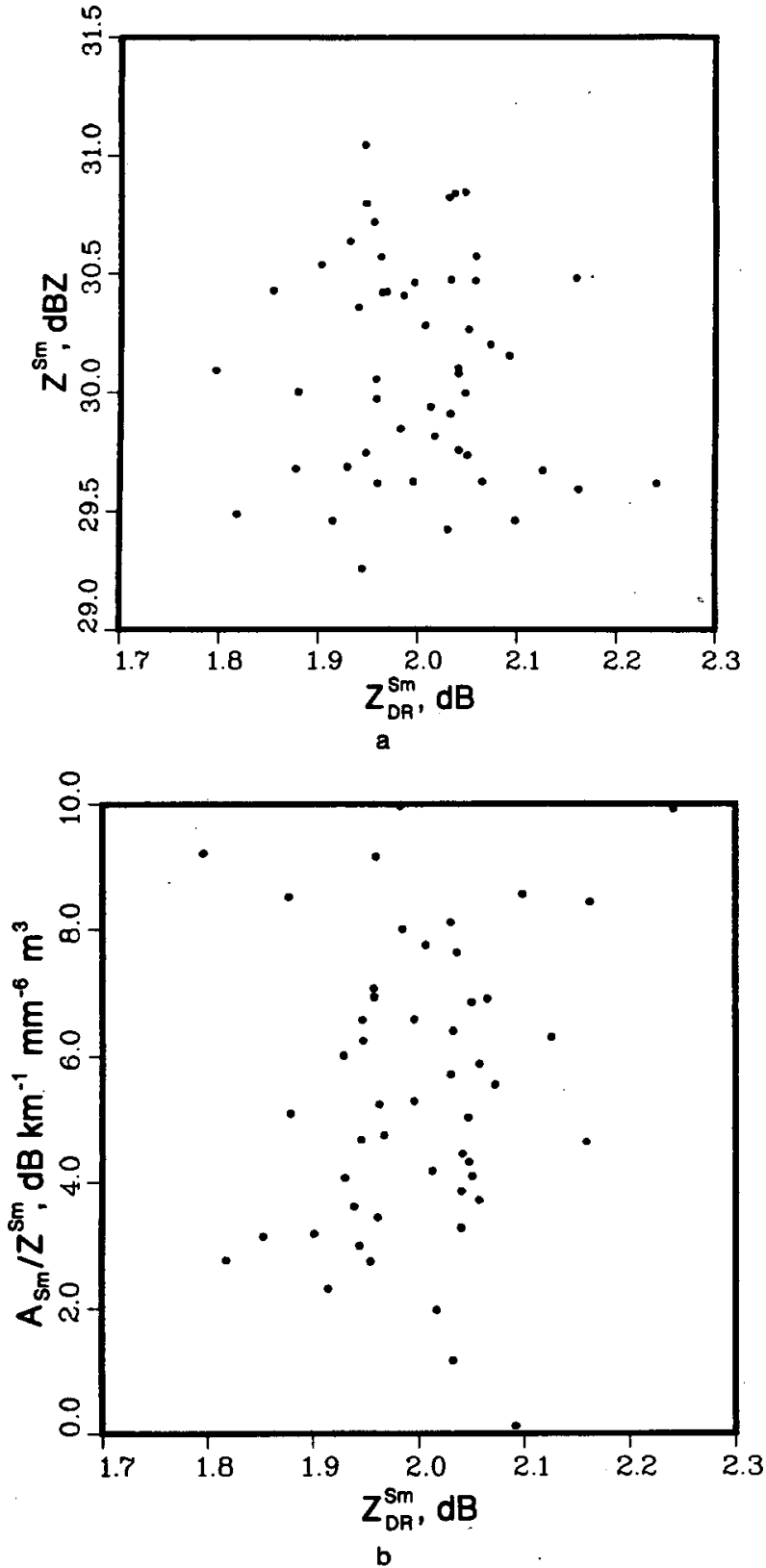

FiG. 4. a. Scatter plot of $Z^{\mathrm{sm}}$ versus $Z_{\mathrm{DR}}^{\mathrm{sm}}$ based on radar simulations, the mean values being $30 \mathrm{dBZ}$ and $2 \mathrm{~dB}$, respectively. The various points represent measurement errors about the mean values. Note that the measurement fluctuations are nearly uncorrelated; see also Table 1. b. As in Fig. 4a except the ordinate is $\left(A^{\mathrm{sm}} / Z^{\mathrm{sm}}\right)$ with mean value $5 \times 10^{-4} \mathrm{~dB} \mathrm{~km}^{-1} \mathrm{~mm}^{-6} \mathrm{~m}^{3}$. Ordinate values are multiplied by $10^{4}$. Note that the measurement fluctuations are nearly uncorrelated.

We now define the following simulation variables:

$Z^{\text {sm }} \quad$ Simulated S-band reflectivity.

$Z_{\mathrm{DR}}^{\mathrm{sm}} \quad$ Simulated S-band differential reflectivity.

$A^{\text {sm }} \quad$ Simulated X-band specific attenuation.

Figure 4 a shows a scatter plot of $Z^{\text {sm }}$ versus $Z_{\mathrm{DR}}^{\mathrm{sm}}$ where the mean values are $30 \mathrm{~dB} Z$ and $2 \mathrm{~dB}$, respec- tively. The various points in the graph show many realizations of $Z$ and $Z_{\mathrm{DR}}$ samples for the same mean values. From the scatter of points in Fig. $4 a$, we can see that $Z$ and $Z_{\mathrm{DR}}$ estimates from the radar are almost uncorrelated. Figure $4 \mathrm{~b}$ shows a scatter plot of $\left(A^{\mathrm{sm}} /\right.$ $\left.Z^{\mathrm{sm}}\right)$ versus $Z_{\mathrm{DR}}^{\mathrm{sm}}$, where the mean values are $5 \times 10^{-4}$ and $2 \mathrm{~dB}$, respectively. Note that the units of $A^{\mathrm{sm}}$ are $\mathrm{dB} \mathrm{km}{ }^{-1}$, while $Z^{\mathrm{sm}}$ is in $\mathrm{mm}^{6} \mathrm{~m}^{-3}$. In addition, this scatter plot does not show any correlation feature, thereby indicating that $\left(A^{\mathrm{sm}} / Z^{\mathrm{sm}}\right)$ and $Z_{\mathrm{DR}}^{\mathrm{sm}}$ are also uncorrelated quantities. The peak fluctuation in $\left(A^{\mathrm{sm}} /\right.$ $Z^{\mathrm{sm}}$ ) covers the range $1 \times 10^{-4}$ to $10^{-3}$ about a mean value of $5 \times 10^{-4}$, with most of the fluctuations contributed by $A^{\mathrm{sm}}$.

The results we have observed here are in direct contrast to the results of section $3 \mathrm{a}$ where we showed that $[A / Z]$ estimates from disdrometer are highly correlated with $Z_{\mathrm{DR}}$. Since the radar $[A / Z]$ relationship is uncorrelated with $Z_{\mathrm{DR}}$ and is also noisy, the actual observation of this relationship using radar measurements would have significantly larger scatter similar to that observed in Fig. 2.

\section{Simulation of $[A / Z]$ and $Z_{\mathrm{DR}}$ estimates from sin- gle / multiple disdrometers}

Simulation of disdrometer-based measurements has been discussed extensively by Chandrasekar and Bringi (1987), and we follow the same procedure here for simulating disdrometer estimates of $A, Z$ and $Z_{\mathrm{DR}}$; see also section 8 of Part I.

We define the following variables:

$Z^{\text {dis }}$ Estimate of reflectivity from simulated disdrometer RSDs.

$Z_{\mathrm{DR}}^{\text {dis }}$ Estimate of differential reflectivity from simulated disdrometer RSDs.

$A^{\text {dis }} \quad$ Estimate of X-band specific attenuation from simulated disdrometer RSDs.

Figure 5 shows a scatter plot of $\left(A^{\text {dis }} / Z^{\text {dis }}\right)$ as a func-

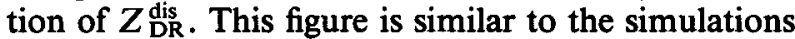
shown in Figs. 6a and $6 \mathrm{~b}$ of Part I. We have chosen a fixed sampling volume of $0.3 \mathrm{~m}^{3}$ to show the features. While we recognize that this sampling volume is small, our conclusions are not significantly altered if larger sampling volumes are used. Figure 5 is obtained by first choosing a particular value of $N_{0}, D_{0}, m$. This gamma RSD is assumed to be sampled by the disdrometer from which $A^{\text {dis }}, Z^{\text {dis }}$ and $Z_{\mathrm{DR}}^{\text {dis }}$ are computed. The values of $N_{0}, D_{0}, m$ are then varied over the full range found in rainfall Ulbrich (1983). Thus, Fig. 5 includes measurement errors as well as natural fluctuations in the gamma parameters. Comparing the simulations in Fig. 5 with the experimental data in Fig. 1a we can see qualitatively the compression in scatter between the $(A / Z)$ versus $Z_{\mathrm{DR}}$ relation, which is caused by the fact that the measurement errors or fluctuations are correlated when 


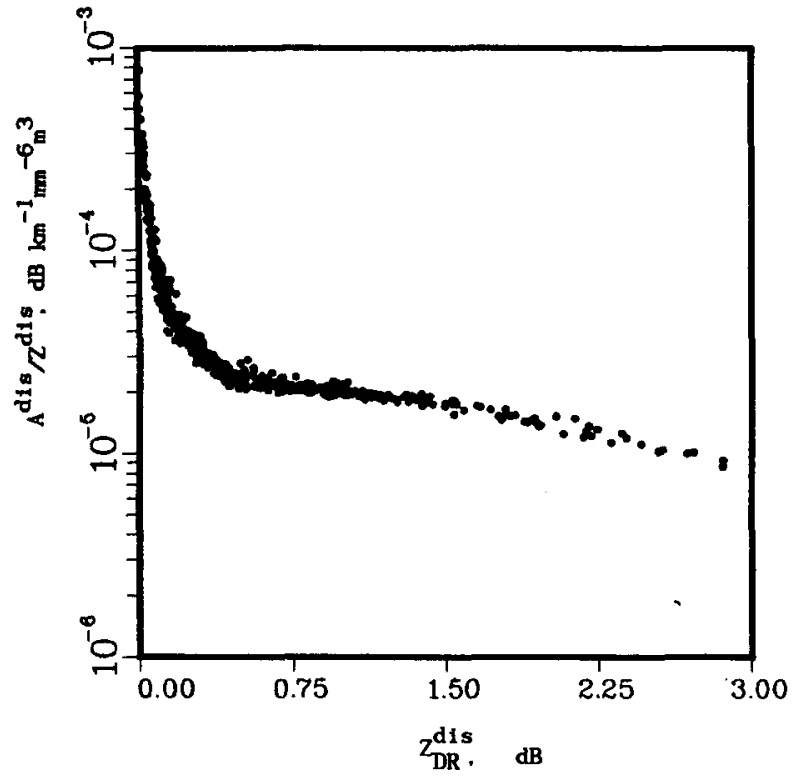

FIG. 5. Scatter plot of $A^{\text {dis }} / Z^{\text {dis }}$ versus $Z$ dis . The disdrometer sample volume is $0.3 \mathrm{~m}^{3}$. Simulations include both measurement errors and natural rainfall fluctuations. The gamma RSD parameters are varied over a wide range.

$A, Z$, and $Z_{\mathrm{DR}}$ are derived from the same disdrometer samples, as was also shown analytically by (7).

To show this explicitly, we have considered a threedisdrometer "experiment" in which each of the triplet $\left(A, Z, Z_{\mathrm{DR}}\right)$ is computed from one of the disdrometers, e.g., $A$ is computed from disdrometer $1, Z$ from disdrometer 2 and $Z_{\mathrm{DR}}$ from disdrometer 3 . The three disdrometers are assumed to sample the same mean rainfall. We assume a fixed sampling volume of 0.3 $\mathrm{m}^{3}$ for each disdrometer. Again, our conclusions would not be altered if larger sampling volumes were used. As before, we vary $N_{0}, D_{0}, m$ over a wide range, representative of natural rainfall. Figure 6 shows the results of this simulation, which can be compared to Fig. 5 . The scatter in Fig. 6 between $(A / Z)$ and $Z_{\mathrm{DR}}$ is now more realistic of the true scatter, which, of course, can be reduced by increasing the sampling volume of each disdrometer but not to the level shown in Fig. 5 .

It is now clear that the experimental data shown in Figs. $1 \mathrm{a}$ and $1 \mathrm{~b}$ can be compared with one another provided due consideration is given to the correlation between $(A / Z)$ and $Z_{\mathrm{DR}}$. The scatter in Fig. 1a is low, while the scatter in Fig. $1 \mathrm{~b}$ is well within the bounds determined by radar signal fluctuations and natural rainfall variations. These comments also apply to the "simulations" of Atlas and Ulbrich (1977), Ulbrich and Atlas (1984), and Atlas et al. (1984), who compute subsets of $A, Z$, and $Z_{\mathrm{DR}}$ from disdrometer RSD samples. The dramatic reduction in scatter they obtain when going from single to multiparameter methods is also aided by statistical correlations. The main reason behind the high correlation is that $A, Z$, and $Z_{\mathrm{DR}}$ (as well as other moments of the RSD) are calculated using their definitions, i.e., integrals or moments of the RSD. This is convenient, since no a priori assumption need be made about the form of the RSD; one just accepts the disdrometer samples. The drawback of course is that the measurement errors are correlated if the same disdrometer data is used to compute all the variables. One alternative to using multiple disdrometers is to use different types of estimators for the variables [see, e.g., Wong and Chidambaram (1985)], in which case the estimators may have a lesser chance of being correlated (this would need to be checked). The drawback is that one makes an a priori assumption about the form of the RSD, e.g., the gamma form. On the other hand, the reduced scatter of Fig. 1a due to correlations clearly shows that there exists a mean relationship between $[A / Z]$ and $Z_{\mathrm{DR}}$ which can be derived from disdrometer samples of natural rainfall without the need to assume a particular form of the RSD.

\section{Conclusions}

The fluctuations of radar and disdrometer measurements of rainfall are controlled by two different stochastic processes. We have shown that a unique relationship exists between $[A / Z]$ and $Z_{\mathrm{DR}}$ for gamma RSDs. Disdrometer estimates of $[A / Z]$ are highly correlated with $Z_{\mathrm{DR}}$, and we have obtained an analytic expression for the correlation coefficient, which shows that the correlation is negative, with typical value of -0.9 . We have also shown analytically that for the case

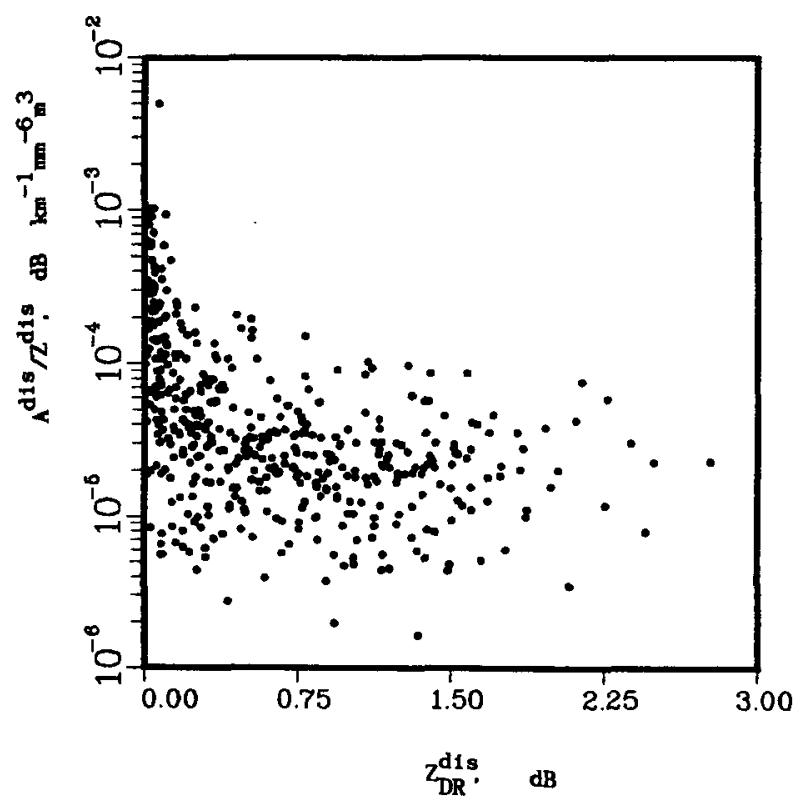

FIG. 6. As in Fig. 5 except that $A^{\text {dis }}, Z^{\text {dis }}$ and $Z$ dis are computed from three different disdrometers all of which sample the same mean gamma RSD parameters. The sample volume is $0.3 \mathrm{~m}^{3}$. 
of a logarithmic receiver, the correlation between $Z$ and $Z_{\mathrm{DR}}$ estimates from radar typically takes values of the order 0.08 . We emphasize that these correlations are due to measurement errors. Since there are three radar measurables involved in the observation of the $[A / Z]$ versus $Z_{\mathrm{DR}}$ relationship, the actual radar data will have a lot of scatter around the mean relationship. The fluctuations in the measurements of $A, Z$ and $Z_{\mathrm{DR}}$ contribute cumulatively to the scatter if the measurements are uncorrelated, as happens with radar observables. In particular, the measurement of attenuation is very noisy when obtained by the dual-frequency ratio technique (Eccles and Mueller 1973; Tuttle and Rinehart 1983). But in the case of disdrometer, the fluctuations of $[A / Z]$ are negatively correlated with $Z_{\mathrm{DR}}$ with a high degree of correlation. The mean value of $[A / Z]$ also decreases with increasing mean $Z_{\mathrm{DR}}$. Thus the correlation helps in reducing the scatter and shows the mean relationship clearly. To explicitly show that this feature is due to statistical correlations, we have simulated $A, Z$, and $Z_{\mathrm{DR}}$ measurements from three independent disdrometers each measuring one of the variables. The $[A / Z]$ versus $Z_{\mathrm{DR}}$ plots show negligible scatter when using a single disdrometer as compared to that when using three disdrometers, although all of them had the same sample volume.

Acknowledgments. This research was supported by the U.S. Army Research Office. Simulations were performed on Colorado State University's Cyber 205 supercomputer through a grant from the Institute of Computational Studies. One of the authors (VC) is a fellow of this Institute.

\section{REFERENCES}

Atlas, D., and C. W. Ulbrich, 1974: The physical basis for attenuationrainfall relationships and the measurement of rainfall parameters by combined attenuation and radar methods. J. Rech. Atmos., 8, 257-298.

$\longrightarrow$, and — 1977: Path- and area-integrated rainfall measurement by microwave attenuation in the $1-3 \mathrm{~cm}$ band. J. Appl. Meteor., 16, 1322-1331.

-, and R. Meneghini, 1984: The multiparameter remote measurement of rainfall. Radio Sci., 19, 3-22.
Aydin, K., T. A. Seliga, C. P. Cato and M. Arai, 1983: Comparison of measured X-band reflectivity factors with those derived from S-band measurements at horizontal and vertical polarizations. Proc., 21st Conf. on Radar Meteor., Edmonton, Alberta, Amer. Meteor. Soc., 513-517.

Bringi, V. N., T. A. Seliga and S. M. Cherry, 1983: Statistical properties of the dual-polarization differential reflectivity $\left(Z_{\mathrm{DR}}\right)$ radar signal. IEEE Trans. Geosci. Remote Sensing, 21, 215-220.

_, V. Chandrasekar and J. Vivekanandan, 1986: X-Band attenuation studies in convective rainshafts using multiparameter radar. $J$. Wave-Material Interaction, 1, 117-140.

Brockwell, P. J., and R. A. Davis, 1986: Time Series: Theory and Applications. Springer-Verlag, 519 pp.

Chandrasekar, V., and V. N. Bringi, 1987: Simulation of radar reflectivity and surface measurements of rainfall. J. Atmos. Ocean. Tech., 4, 464-478.

$\longrightarrow,-$ and P. J. Brockwell, 1986: Statistical properties of dualpolarized radar signals. Preprints, 23rd Conf. on Radar Meteorology, Snowmass, Amer. Meteor. Soc., 193-196.

Eccles, P. J., and E. A. Mueller, 1973: X-band attenuation and liquid water content estimation by a dual-wavelength radar. J. Appl. Meteor., 10, 1252-1259.

Goddard, J. W. F., and S. M. Cherry, 1984: The ability of dual polarization radar (co-polar linear) to predict rainfall rate and microwave attenuation. Radio Sci., 19, 201-208.

Jameson, A. R., 1983: Microphysical interpretation of multiparameter radar measurements in rain. Part II: Estimation of raindrop distribution parameters by combined dual-wavelength and polarization measurements. J. Atmos. Sci., 40, 1803-1813.

Joss, V. J., and A. Waldvogel, 1969: Raindrop size distribution and sampling size errors. J. Atmos. Sci., 26, 566-569.

Leitao, M. J., and P. A. Watson, 1984: Application of dual linearly polarized radar data to prediction of microwave path attenuation at 10-30 GHz. Radio Sci., 19, 209-221.

Mood, M. A., F. A. Graybill and D. C. Boes, 1974: Introduction to the Theory of Statistics. McGraw-Hill, $564 \mathrm{pp}$.

Oguchi, T., 1983: Electromagnetic wave propagation and scattering in rain and other hydrometeors. Proc. IEEE, 71, 1029-1078.

Tuttle, J. D., and R. E. Rinehart, 1983: Attenuation correction in dual-wavelength analyses. J. Climate Appl. Meteor., 22, 19141921.

Ulbrich, C. W., 1983: Natural variations in the analytical form of the raindrop size distribution. J. Climate Appl. Meteor., 22, $1764-1775$.

- and D. Atlas, 1984: Assessment of the contribution of differential polarization to improved rainfall measurements. Radio Sci., 19, 49-57.

Wong, R. K. W., and N. Chidambaram, 1985: Gamma size distribution and stochastic sampling errors. J. Climate App. Meteor., 24, 568-579.

Zrnić, D. S., 1975: Simulation of weatherlike Doppler spectra and signals. J. Appl. Meteor., 14, 619-620. 